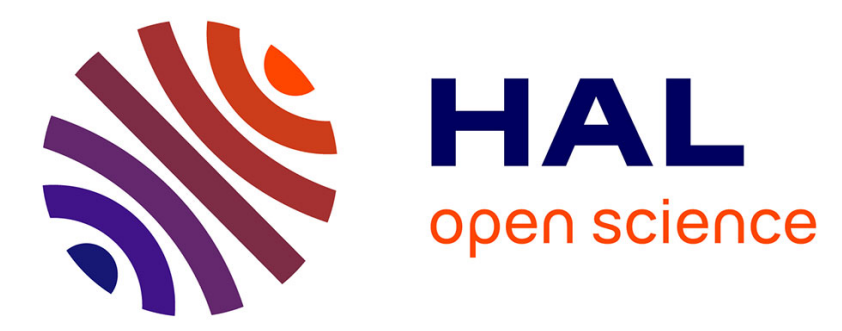

\title{
L'identification et le corps du migrant : comment laisser des traces de présence physique dans un monde numérique
}

Claire Scopsi

\section{- To cite this version:}

Claire Scopsi. L'identification et le corps du migrant: comment laisser des traces de présence physique dans un monde numérique. Béatrice GALINON-MÉLÉNEC. L'Homme-trace: Des traces du corps au corps-trace, CNRS Editions, 2017, 978-2-271-11417-4. 10.4000/books.editionscnrs.29947 . hal01967959

\section{HAL Id: hal-01967959 \\ https://hal.science/hal-01967959}

Submitted on 1 Jan 2019

HAL is a multi-disciplinary open access archive for the deposit and dissemination of scientific research documents, whether they are published or not. The documents may come from teaching and research institutions in France or abroad, or from public or private research centers.
L'archive ouverte pluridisciplinaire HAL, est destinée au dépôt et à la diffusion de documents scientifiques de niveau recherche, publiés ou non, émanant des établissements d'enseignement et de recherche français ou étrangers, des laboratoires publics ou privés. 


\title{
L'identification et le corps du migrant : comment
}

\section{laisser des traces de présence physique dans un}

\section{monde numérique}

\author{
Claire Scopsi
}

Le corps de l'étranger est l'objet d'une attention particulière de l'administration car le nomade nous dirions aujourd'hui l'individu en mobilité - est celui dont aucun témoin présent ne peut garantir l'identité. Il faut alors s'appuyer sur des documents émanant de sources fiables de la région dont il est issu, mais ces documents peuvent être mensongers, falsifiés ou volés. Il ne reste alors pas d'autre solution que d'interroger le corps. Au corps de l'étranger, l'administration demande des faits, des dates, des indices et des preuves. Elle l'analyse, le code, lui applique des marques et des métadonnées, en extrait des références numériques et, enfin, le valide. Ce corps est un document.

\section{INTRODUCTION : TRACES CORPORELLES ET DOCUMENTALITE DU CORPS}

Les Journées «Traces du corps » des 30 juin et $1^{\text {er }}$ juillet 2016 m'ont offert l'opportunité de lier cette réflexion sur la documentalité du corps à celles des nombreux contributeurs des ouvrages collectifs publiés par l'équipe Homme-Trace. J'y ai répondu avec intérêt pour plusieurs raisons. Il est tout d'abord toujours excitant d'expérimenter l'application d'un concept exogène à un objet d'étude familier - ici les migrants en situation irrégulière et leur processus de régularisation. L'exercice, au départ formel, s'est révélé fructueux. Le paradigme de l'Homme Trace, tel qu'il est exprimé par Béatrice Galinon-Mélénec, c'est dire comme un système d'interactions de signestraces, certains reçus de l'environnement et intégrés par le corps, d'autres produits, rejetés pour ainsi dire dans l'environnement, a permis de rassembler et d'ordonner des procédés 
d'identification de l'étranger qui m'apparaissaient disparates et anachroniques (d'Homère au Big data !) et de faire émerger des logiques immuables, une quasi universalité des usages des traces corporelles par les institutions identifiantes.

J'avais aussi pour projet d'apporter un complément au volet «criminologique » des travaux d'Homme-Trace. L'exemple de l'enquête policière déductive, que la culture populaire, de Sherlock Holmes aux «Experts» des séries télévisées nous rendent familières, y est parfois évoqué pour définir la fonction indicielle de la trace ${ }^{1}$. Je voulais montrer l'autre volet de la trace dans les pratiques policières, celui, moins visible, des fichiers administratifs, du travail documentaire. Relever des empreintes sur une scène de crime ne sert à rien s'il n'existe des fichiers préalablement constitués pour les comparer. Ces données accumulées, doivent, pour être toujours plus efficaces, être toujours plus volumineuses. C'est ce qui conduit les sociétés à glisser inévitablement de l'identification à la surveillance.

Enfin, ce texte est à relier aux articles du Livre III du Tome 1 de l'Homme-Trace publié en 2011 et consacré aux « sans-trace » (Bernardot 2011) et plus particulièrement celui de Gino Gramaccia sur « Les institutions de la médiation symbolique en quête de l'Homme-trace » (2011). Minorités, colonisés, précaires sont l'objet d'une guerre des traces. Les traces qu'ils produisent, marque de leur présence intrusive dans des espaces qui ne sont pas faits pour eux, sont effacées, doivent être effacées par les institutions en tant que témoignage d'un déséquilibre, d'un désordre à gérer.

Comment alors retracer l'existence des sans-trace ? Je voulais ici revenir sur cette relation entre la trace des migrants en situation irrégulière et les institutions médiatrices (ici les préfectures) dont le rôle symbolique et sacré d'institution républicaine cède peu à peu, selon Gramaccia, à la logique gestionnaire, passant de la vision d'un destin national au bon gouvernement de l'usager.

Les espaces des migrants en situation irrégulière, sont ceux de la non-trace, donc de l'invisibilité. Ne pas se faire voir, se fondre dans la société, ne pas dire, ni au travail, ni à l'école. Pourtant les traces jaillissent sur les bords, dans les camps de migrants de Calais, Vintimille, ou Lampedusa,

\footnotetext{
${ }^{1}$ Béatrice Galinon Mélénec convoque fugacement Sherlock Holmes dans le Tome 1 (2011, p.172) et plus précisément l'enquête su la mort de Pasolini dans le Tome 3 (2015, p.11) pour définir les notions de traces, identités et empreintes.
} 
dans les files d'attente des Centre de Réception des Etrangers, ou de l'OFPRA, dans les aéroports, bref, dans les lieux de déséquilibre des flux, là où l'institution empêche d'entrer ou force à partir. Les migrants deviennent visibles et médiatisables sous la forme d'une « crise ». Ailleurs, dans les espaces d'invisibilité où les migrants circulent, travaillent, s'intègrent, la trace joue un rôle moins spectaculaire mais plus insidieux, mis en scène par les institutions policières. Il s'agit de les analyser pour en déduire les motifs d'un refus ou d'une acceptation à résidence. Pas de violence physique à noter dans les espaces de non-trace, contrairement aux bordures , même par le passé, si l'on excepte la marque au $\mathrm{fer}^{2}$, mais un usage violent du physique de la part de l'institution gestionnaire. Car n'est ce pas une violence sociale, tout autant que l'effacement des traces, que l'instrumentalisation des traces du corps de l'autre pour l'exclure, et le renvoyer à son désespoir ? Ce travail sur le corps-document résulte de ma pratique, depuis huit ans, au sein d'une permanence du Réseau Education Sans Frontières située dans le quartier de la Goutte d'Or à Paris, dans laquelle des bénévoles aident les migrants ${ }^{3}$ en situation illégale à mener leurs démarches de régularisation. Plus d'une centaine de dossiers m'ont familiarisée avec les méthodes des préfectures. Je croise cette expérience avec les travaux menés dans le cadre du collectif OUDIPO (ouvroir de diplomatique potentielle), où des chercheurs et des professionnels des archives et de la documentation technique s'interrogent sur l'application des méthodes diplomatiques aux documents numériques. La diplomatique est une discipline, formalisée il y a plusieurs siècles, qui définit un vocabulaire et une méthode pour critiquer l'authenticité et la fiabilité des documents écrits. Elle est traditionnellement appliquée aux archives dans le cadre de la recherche de la vérité historique ainsi que dans toute situation administrative ou judiciaire « quand le nœud du litige ou

\footnotetext{
${ }^{2}$ Marque au fer des récidivistes, que les sommiers de Bertillon avaient pour objectif de remplacer par une méthode d'identification moins intrusive et douloureuse.

${ }^{3}$ J'utilise le terme, pourtant controversé, de migrant pour souligner le caractère inachevé du processus d'immigration des personnes qui n'ayant pas encore obtenu de titre de séjour, ne peuvent accéder à certains droits - travail déclaré, études et diplômes supérieurs, aides sociales - et vivent sous le risque permanent d'une expulsion du territoire. Dénué de toute signification juridique, ce terme permet de mettre l'accent sur un temps particulier de la mobilité, la migrance, situé entre la rupture du départ et l'achèvement du processus par l'intégration.
} 
de la polémique est constitué par un document ou une série de documents contestés ${ }^{4} »$. Le point de jonction entre ces deux univers se situe dans la question de la fraude documentaire. Lorsque le migrant est suspecté par l'autorité administrative de mentir sur sa situation, son origine et son identité, c'est à son corps de rétablir la vérité.

Dans la première partie de ce texte, je reviendrai sur les origines de l'identité administrative et sa relation avec les situations de mobilité, en rappelant les trois procédés fondamentaux que nous retrouvons tout au long de l'histoire de la surveillance : l'identification «par ce que l'on est », l'identification «par ce que l'on porte » et l'identification «par ce que l'on sait». Dans la deuxième partie je développerai le concept de corps-document en montrant comment, à l'époque moderne, le corps est l'objet de traitements - référencement, classement, description, indexation ou décodage - qui relèvent d'une documentalité. Il m'arrivera au cours de cette partie de m'éloigner de la notion stricte de trace car le concept de corps-document n'est pas exactement superposable à celui d'homme-trace, mais j'y reviendrai dans la troisième partie consacrée aux contradictions entre les logiques physiques et juridiques, pour montrer qu'à l'époque de l'eadministration, les pratiques tendent à délaisser les preuves par le corps, et privilégient les preuves administratives, sans toutefois en exploiter les data.

Ce travail emprunte à plusieurs réflexions: celle de Michel Foucault, sur l'investissement corporel du pouvoir et l'invention d'une « anatomie politique », même si « Surveiller et Punir » (1975), s'intéresse à la discipline et la punition sans aborder l'identification. Il doit bien sûr aux travaux de Gérard Noiriel sur l'immigration et l'identité nationale (1992, 2007), à ceux d'Armand Mattelart sur la surveillance globalisée (2007) et bien sûr à ceux de Béatrice Galinon-Mélénec pour les concepts de trace, d'indice et d'empreinte. Quant aux menaces de contrôle par les data du web, qui relèvent plus dans ce cas précis du fantasme que de la réalité, ils puisent dans les notions de data-panoptisme de Sadin (2015) et d'Etat prédictif de Viktor Mayer-Schönberger et Kenneth

\footnotetext{
${ }^{4}$ TESSIER (G.), Diplomatique, in L’Histoire et ses méthodes, Encyclopédie de la Pléiade 1961
} 
L'identification et le corps du migrant

Cukier (2014). Enfin il faut signaler l'apport du portail Crimino-corpus ${ }^{5}$, du Centre pour les humanités numériques et l'histoire de la justice, enthousiasmante mine de ressources scientifiques et de fac-simile de documents anciens.

\section{L'INDIVIDU EN COMMUNAUTE ET L'INDIVIDU EN MOBILITE}

Si l'exemple des migrants est éloquent, c'est que la situation de mobilité illustre la problématique originelle de l'identité administrative : il s'agit de reconnaitre et tracer celui qui vient d'ailleurs qu'il soit voyageur, réfugié ou immigré. Pour Gérard Noiriel « Les moments de crise apparaissent quand il existe un important décalage entre le niveau de mobilité et l'état de la technologie identificatrice » (Noiriel, 1993, p. 8). Les moments d'intense mobilité, périodes de guerres, d'épidémies, de catastrophes naturelles, de crises économiques qui jettent les populations sur les routes sont aussi des périodes d'innovation des méthodes de surveillance. Mais quels que soient les procédés et les technologies mis en œuvre, on peut les réduire à trois fondamentaux qui, séparément ou combinées, permettent d'identifier un individu. 1) ce qu'il est: son apparence physique, 2) ce qu'il sait : un fait, un détail que seul l'individu qu'il affirme être peut connaitre et enfin 3) ce qu'il porte : un objet spécifique et discriminant qu'il détient de façon exclusive et peut produire à tout moment.

\section{L'identification par « ce que l'on est»}

Dans les sociétés traditionnelles l'identité est attestée par la communauté directe, ceux avec qui un individu est en contact quotidien, qui connaissent son lignage et qui ont suivi l'évolution de son apparence physique. Cette identité par le « face à face », antérieure au droit écrit, n’a pas disparu en droit contemporain qui peut requérir, lors de certains actes notariaux, la présence de deux témoins capables de garantir par la notoriété l'authenticité d'une filiation. Cette forme d'identification par «ce que l'on est » présente l'inconvénient de résister très difficilement aux ruptures liées aux mobilités et au temps. La situation, est si dérangeante qu'elle est au cœur de

\footnotetext{
${ }^{5}$ https://criminocorpus.org/
} 
plusieurs mythes grecs : Edipe, à son corps défendant, et Ulysse, par ruse, incarnent la figure du voyageur ou du mendiant, dont le visage changé masque l'identité. De célèbres affaires illustrent la difficulté pour une communauté de reconnaitre à coup sûr celui qui, absent depuis de longues années, revient vieilli et changé. L'affaire « Martin Guerre », dont l'arrêt est rendu au parlement de Toulouse en 1560 et au cours de laquelle près de 300 témoins interviennent, est devenu emblématique du cas d'usurpation d'identité. Le traité qu'en a tiré Jean de Coras, livre les réflexions méthodologiques des juges : un signe corporel distinctif «comme des verrues des mains, gouttes de sang à l'œil, et enfoncement de l'ongle » n'est preuve d'identité que si il est évoqué par plus d'un témoin tandis que plusieurs témoins reconnaissant chacun un signe différent, ne peuvent attester d'une identité certaine : « ce sont témoins singuliers qui ne sont preuve encore qu'ils fussent mille déposants chacun de son fait. » (Jean de Coras, 1560, p. 79).

\section{L'identification par « ce que l'on porte »}

Lorsque l'intéressé est exogène à la communauté, il devient impossible de l'identifier au moyen du face à face, on recourt alors à un tiers distant. Depuis l'antiquité, les voyageurs sont porteurs d'une lettre de recommandation écrite d'un personnage digne de foi (prince, prêtre ou notable) ou d'un sumbolon, objet brisé en deux parties, parfois transmis aux générations suivantes, dont les deux parties rassemblées servent de signe de reconnaissance. Cette pratique est encore vivace : le principe du sumbolon a inspiré les plaques militaires ou « dog tag », portées au cou par le soldat de la seconde guerre mondiale. Prédécoupées, elles sont composées de deux éléments portant les mêmes informations et notamment un numéro de sécurité sociale. L'un des deux éléments demeurait sur le corps du soldat mort ou blessé, l'autre était rapporté au camp et permettait d'évaluer les pertes. Le principe du sumbolon est plastique et l'on peut considérer que tout système d'identification composé de deux éléments associés par un identifiant unique dont l'un au moins doit être porté sur soi en est l'héritier. C'est le cas des cartes d'identités, passeports, dont le numéro renvoie à un fichier et que l'on doit porter ou pouvoir produire en cas de contrôle. Mais le procédé ne garantit pas absolument l'identité du porteur, car la lettre ou l'objet peut être dérobé. 


\section{L'identification « par ce que l'on sait » :}

Il y a certains faits que seul un très petit nombre d'individus peut connaitre. Ici, ce n'est pas la nature même de la connaissance qui compte, le sens qu'elle véhicule, mais sa spécificité ; ainsi Pénélope reconnait-elle Ulysse, malgré son déguisement, par la description qu'il livre de leur couche nuptiale, qu'il a bâtie de ses propres mains (Odyssée, chant XXIII). Au début du 19e siècle, Karl-Wilhelm Naundorff, horloger prussien, affirme être le dauphin Louis XVII et est reconnu par de nombreux témoins proches de la famille royale, alimentant le mythe de la survivance de l'enfant du Temple. L'âge de Naundorff (il a une vingtaine d'années lorsqu'il se manifeste), la fantaisie des gravures représentant Louis XVII ne permet pas le rapprochement par le corps. Mais en livrant des détails intimes sur l'enfance de Louis XVII, Naundorff parvient à convaincre Agathe de Rambaud qui avait occupé la charge de «berceuse du Dauphin ». Les plus récents travaux de comparaison de l'ADN des restes du Dauphin et de ceux de Naundorff montrent qu'il ne peut être l'enfant du Temple, cependant, la « berceuse du Dauphin » resta toute sa vie convaincue d'avoir retrouvé l'héritier du trône de France. Le recours au procédé du «ce que l'on sait » est fréquent aujourd'hui sous la forme du mot de passe ou de la question test ${ }^{6}$. Mais un mot de passe, une anecdote secrète, peuvent être divulgués, dérobés par ruse ou indiscrétion. L’efficacité du procédé dépend du maintien du secret.

La fragilité des trois procédés conduit à les superposer pour augmenter leur fiabilité et des procédés combinant «ce que l'on est» et «ce que l'on porte» sont élaborés. Ils consistent à associer une description physique du sujet au document qu'il porte sur lui, pour montrer qu'il n'a pas été dérobé. C'est bien le cas de nos cartes d'identité et de nos passeports, qui, outre le fait d'être rangés dans nos poches, sont revêtus de notre photographie.

Je reviens donc dans la seconde partie sur les utilisations du corps (« ce que l'on est ») dans les divers systèmes d'identification pour montrer la construction d'une documentalité du corps.

\footnotetext{
${ }^{6}$ La société de transferts d'argent Western Union utilise une « question test » lorsque le bénéficiaire du transfert ne dispose pas de pièce d'identité. Il peut s'agir par exemple du nom de jeune fille de la mère, qui ne figure pas sur les documents officiels mais est supposé être connu du bénéficiaire.
} 


\section{LE CORPS-DOCUMENT}

L'évolution des protocoles d'identification se produit souvent à des moments de déséquilibre, de danger ou d'incertitude entrainant un accroissement des circulations. A la mort de Louis XIV, la démobilisation des troupes et les désertions conduisent à l'ordonnance du 2 juillet 1716 qui instaure les registres de contrôle des troupes. Chaque régiment inscrit ses hommes dans ce registre, en y associant une courte description physique. Un soldat démobilisé ou en permission doit porter sur lui un document portant cette description et l'attestation de son régiment, faute de quoi il est considéré déserteur. Le système des registres s'étend par la suite à d'autres populations de voyageurs ou de vagabonds et les aubergistes, les communautés de métier, les prêtres sont aussi habilités à établir ces «permissions». A l'issue de la Révolution Française, la jeune république étend les registres à toute la population.

\section{La production de métadonnées corporelles}

Mais les descriptions physiques apposées au document restent sommaires, fantaisistes et subjectives $^{7}$, rendant difficile le rapprochement avec le corps réel et le besoin se fait sentir d'un signalement plus rationnel. Les catégories (visage, front, yeux, nez, bouche, menton) et les qualificatifs (« fort », « allongé », « moyen ») se standardisent. (Gutton, 1973, Denis, 2000). Cette éducation des personnels administratifs, s'opère lentement tout au long du $19^{\mathrm{e}}$ siècle pour aboutir en 1895 au « cours de signalements et de reconnaissances anthropométriques » dispensé aux agents de police, devenu obligatoire et sanctionné par un «brevet d'étude du signalement descriptif » en 1902 (About, 2004). Ce signalement, également nommé « portrait parlé », fleuron du système Bertillon, consiste en une centaine d'annotations, reposant sur un lexique de termes conventionnels.

\footnotetext{
${ }^{7}$ Un exemple de description non normalisée : dans les copies de registres de sortie des mendiants de la Charité de SaintEtienne, figurant dans la thèse de Jean-Pierre Gutton, on lit en 1724 : «André la Guimonde, âgé de vingt-neuf ans, d'Abbeville en Picardie, frotteur, haut de cinq pieds deux pouces, gros, cheveux châtains, sourcils de même \& épais, les yeux noirs \& grands, la bouche un peu tournée, le nez gros \& boutonné, le visage plein et rouge, un doigt de la main gauche plus court que les autres. »(Guitton, 1973, p. 110).
} 
L'identification et le corps du migrant

\section{Le corps classifié}

Le portrait parlé ouvre la voie aux fichiers corporels classifiés qui permettront d'accéder à l'identité d'un individu à partir de ses caractéristiques physiques. Jusqu'aux profondes modifications de Bertillon à partir de 1882, les fichiers de description judiciaires sont, certes, centralisés, mais accessibles seulement par le nom de l'individu. Ce classement, sensible aux erreurs ou variations de graphie et aux mensonges des prévenus est assez peu efficace. Les sommiers judiciaires de Bertillon consistent en un index des noms phonétique, donc moins sensible aux graphies multiples ${ }^{8}$, et un système de renvoi entre les noms et les sobriquets. S'y ajoute un référentiel inspiré de l'application des méthodes statistiques au corps humain de Quetelet $^{9}$ (Piazza, 2014). Une classification ingénieuse des mesures permet d'accéder à la photographie et à la fiche d'un individu.

\section{La mesure et l'empreinte}

L'analyse qualitative du corps, selon l'expression même de Bertillon ${ }^{10}$, supplante la reconnaissance formelle du visage et du corps. La mise en chiffres devient obligatoire dans la circulaire du 25 août 1893 : « tout détenu, prévenu ou condamné doit être mesuré ; c'est-à-dire qu'il en est ainsi de tous les individus écroués pour lesquels on se contentait antérieurement d'un signalement descriptif. »

\footnotetext{
${ }^{8}$ Source : Notice sur l'organisation et le fonctionnement du service des sommiers judiciaires. Classement phonétique, Service régional de l'identité judiciaire de la préfecture de police de Paris, 1889 https://criminocorpus.org/fr/bibliotheque/page/89753/\#page

${ }^{9}$ Sont mesurés : la taille, la longueur comprise entre l'épaule gauche et le majeur droit, le buste, la longueur de la tête du crâne au front, la largeur de la tête de la tempe gauche à la tempe droite, la longueur du médius gauche, la longueur de la coudée gauche. Chacune de ses mesures, qualifiée de grande, moyenne ou petite, permet de progresser dans la classification, jusqu'à l'identification d'un individu.

${ }^{10}$ « Les récidivistes auront donc toujours un intérêt immédiat pour échapper à cette graduation de la correction, à tromper, à changer de noms et prénoms. Or, nous avons démontré que nos mensurations jouent pour la reconnaissance des malfaiteurs le rôle d'une véritable analyse qualitative. » Alphonse Bertillon, L'Identité des récidivistes et la loi de relégation, Masson, 1883.
} 
RELEVÉ

$\mathrm{DU}$

SIGNALEMENT ANTHROPOMÉTRIQUE

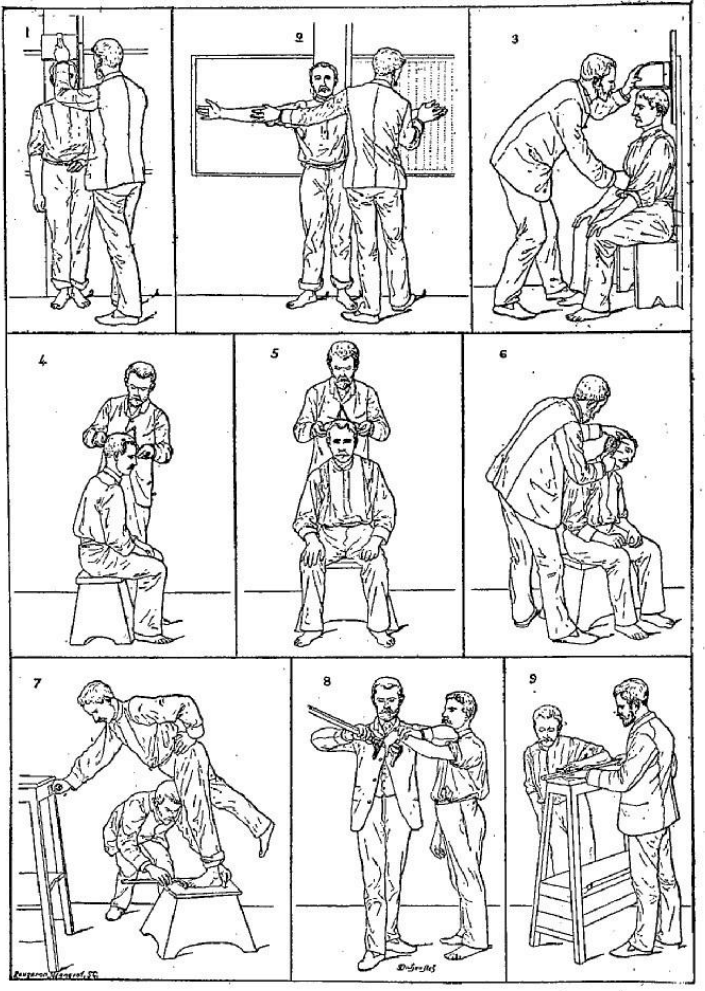

I. Taille. -2 . Envergure. -3 . Buste. -

4. Longueur de la tète. -5 . Largeur de la tête. -6 . Oreille droite. -

7. Pied gauche, -8 . Médius gauche: - 9. Coudée gauche.

Fig.1 Frontispice de L’identification anthropométrique : instructions signalétiques, Alphonse Bertillon, 1893

Mais la reconnaissance de forme n'a pas dit son dernier mot: elle revient en force avec la photographie judiciaire et la dactyloscopie qui supplante le système de mesure osseuse. Car mesurer le corps est une procédure longue, applicable aux détenus et prévenus à disposition de la police, mais malcommode à généraliser à la population en liberté. La captation rapide d'une empreinte $^{11} \mathrm{du}$ corps, par encrage ou photographie, pour l'analyser et la comparer ensuite à une collection s'avère plus généralisable, tout en étant plus fiable que la description visuelle par un agent. Bertillon privilégie cependant la mesure qu'il considère comme « une base d'identification infiniment moins trompeuse que les ressemblances photographiques » (Bertillon, 1886, p. 207) car la physionomie d'un individu varie considérablement entre ses diverses arrestation et selon les

\footnotetext{
${ }^{11}$ Il s'agit bien ici d'une captation, c'est-à-dire d'une trace produite volontairement dont on exploite les caractères distinctifs, qu'il faut distinguer de la trace involontaire, l'empreinte laissée sur une scène de crime
} 
L'identification et le corps du migrant

conditions et méthodes de prise de vue. Pour autant, il reconnait que la forme du profil, du nez, la forme de l'oreille varie peu. Des albums de photographies de détails du corps, oreilles, mains, profils, sont diffusés par les service d'identification judiciaire.

Les deux logiques, la mesure et l'empreinte, rivalisent donc jusqu'au moment où la captation numérique les réconcilie en permettant de coder numériquement les éléments formels de l'empreinte. Mais on peut encore lire cette dichotomie dans les techniques modernes d'identification biométrique. L'examen comparatif de photographies du visage, qu'il soit holistique (la totalité du visage) ou détaillé, superpose la photographie du « candidat » et celle du fichier. Ensuite est comparé l'éloignement plus ou moins grand des contours ; pour une réalisation optimale, il exige que la pose, et l'échelle du cliché soient identiques. L’identification automatique résulte d'un encodage des données faciales (mesures d'espacement, calculs d'angles) qui caractérisent un individu. Cet encodage d'un morceau du corps pourra, dans un avenir relativement proche, être appliqué à la voix, à la thermographie du corps, à la géographie veineuse de la main.

En février 2016, l'organisation des Nations Unies (PAM) a inauguré un système de paiement novateur, qui utilise le scan de l'iris pour permettre aux réfugiés syriens vivant dans des camps jordaniens d'acheter de la nourriture chez des commerçants locaux, plutôt que d'utiliser de l'argent, des coupons alimentaires ou des cartes de crédit ${ }^{12}$. Il s'agit bien ici de remplacer « ce que l'on porte » l'objet, la carte qui justifie d'une droit et qui, dans un contexte troublé, peut être dérobée ou faire l'objet d'un trafic, par « ce que l'on est », un élément corporel inaliénable.

\section{La marque et l'indice}

Le contexte joue un rôle important dans les modes d'usages des corps pour l'identification. L'environnement légal, l'enjeu de l'identification, l'époque, l'amélioration des connaissances techniques ou documentaires orientent le choix des protocoles. Selon qu'on recherche, au 18eme siècle, un récidiviste pour l'envoyer aux galères, ou, aujourd'hui, un mineur isolé étranger pour le

\footnotetext{
${ }^{12} \mathrm{Cf}$. « Le PAM annonce une innovation permettant aux réfugiés syriens de payer leurs achats « en un clin d'œil » Centre d'actualité de l'ONU, 17 février 2016, http://www.un.org/apps/newsFr/storyF.asp?NewsID=36653 (accédé le 12 juin 2016).
} 
confier à l'Aide Sociale à l'Enfance, ou bien qu'on cherche à établir une filiation pour autoriser un regroupement familial, on en appelle au corps de diverses façons. Mais c'est, à chaque fois, en tant qu'indice qu'il est convoqué.

Les cicatrices, les membres amputés, atrophiés, les difformités, les traces de maladies ont toujours figuré dans les descriptions corporelles. Ce sont des marques, c'est à dire des signes originaux ou rares dont la présence confirme une identité et ce ne sont pas en tant que trace, c'est-à-dire « chose qui a été déposée dans le passé et conservée dans le présent 》(Galinon-Mélénec, 2015, p. 11) qu'elles intéressent l'administration, laquelle ne s'occupe guère de leur origine. Elles ne sont qu'un élément de différenciation. Il en va autrement lorsque la marque est appliquée volontairement par l'autorité publique, comme trace, intimement gravée, d'un acte passé ou d'un statut judiciaire. La marque au fer est appliquée aux criminels, aux esclaves. Elle se prolonge dans le tatouage des déportés des camps d'extermination. Le bracelet électronique, « objet que l'on porte » malgré soi, offre une alternative moins douloureuse, mais toujours infamante. Depuis 2015, plusieurs hommes politiques français ont suggéré d'en équiper les demandeurs d'asile afin qu'ils ne disparaissent pas avant la prise de décision ${ }^{13}$. Cette pratique est déjà en vigueur aux Etats-Unis et au Royaume Uni.

Rarement, les marques acquises prennent aussi aux yeux de l'institution identificatrice la valeur d'indices. Selon Béatrice Galinon-Mélénec « toute chose est trace, elle ne devient indice que dans le contexte d'une recherche rationnelle de signification en vue d'établir qu'une situation passée s'est bien déroulée comme l'interprétant l'imagine »(Galinon-Mélénec, 2015, p. 11). Il en va ainsi lorsque Bertillon présente à l'exposition universelle de 1889 un album photographique des stigmates de la main occasionnés par l'exercice des différents petits métiers parisiens. Cals, blessures, amputations témoignent de la dureté de ces travaux ouvriers, mais l'institution n'y recherche que la confirmation du métier exercé par les cadavres de la morgue.

\footnotetext{
${ }^{13}$ Cf. « Hervé Mariton favorable aux bracelets électroniques pour les demandeurs d'asile », site rtl.fr http://www.rtl.fr/actu/politique/herve-mariton-favorable-aux-bracelets-electroniques-pour-les-demandeurs-d-asile-

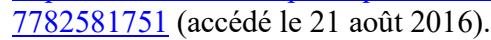


En revanche, les marques et traces innées sont souvent confrontées à des référentiels destinés à faire sens. Longtemps on a associé à la morphologie l'indice ${ }^{14}$ d'un caractère moral. Ici, l'indice ne renvoie pas à un fait passé, comme c'est le cas dans une enquête policière, mais à un possible, une propension à agir d'une certaine façon. La physiognomonie de Johann Kaspar Lavater, puis la phrénologie de Franz Joseph Gall tentent, au 18e siècle, d'établir une relation entre le corps visible et palpable et l'âme intangible. Cesare Lombroso, au 19e siècle, s'appuie sur la statistique pour établir un Atlas des caractéristiques physiques des criminels. La fréquence d'observation des particularités morphologiques chez les criminels, conduit à inférer que la présence de ces particularités est l'indice d'une nature criminelle. Ces théories n'ont plus cours aujourd'hui mais l'inférence persiste dans les contrôles d'identité au faciès. En octobre 2015, l'Etat français est accusé par le défenseur des droits, d'avoir discriminé des citoyens en pratiquant des contrôles ciblés sur des passants noirs et arabes. L'Etat est condamné en première instance et se pourvoie en cassation avec un argument probabiliste : le contrôle visait à identifier des étrangers en situation illégale, et ciblait ceux dont l'apparence physique renvoyait le plus probablement à l'état d'immigré clandestin ${ }^{15}$.

\section{Le corps décodé}

En 2009 la géo génétique a été mise à contribution dans le cadre du «Human provenance pilote project »(HPPP) lancé par l'Agence des Frontières britannique (UK Border Agency). Il s'agit de vérifier par l'ADN mitochondrial la véracité des déclarations d'origine géographique et de nationalité formulées par les demandeurs d'asile. En effet, des candidats à l'asile Kenyan tentaient de se faire passer pour des réfugiés Somaliens, ceux-ci étant prioritaires au droit d'asile. La géogénétique est pratiquée pour retracer les courants migratoires des peuples sur les longs temps

\footnotetext{
${ }^{14}$ Selon Béatrice Galinon -Mélénec « toute chose est trace, elle ne devient indice que dans le contexte d'une recherche rationnelle de signification en vue d'établir qu'une situation passée s'est bien déroulée comme l'interprétant l'imagine » (Galinon-Mélénec, 2015, p. 11). Ici, dans le cas du faciès, l'indice ne renvoie pas à un fait passé, comme c'est le cas dans une enquête policière, mais à un possible, une propension à agir d'une certaine façon.

15 «Condamné pour contrôles au faciès, l'Etat se pourvoit en cassation », Le monde.fr, 16 octobre 2015, http://www.lemonde.fr/police-justice/article/2015/10/16/condamne-pour-controles-au-facies-1-etat-se-pourvoit-encassation 4790793 1653578.html (accédé le 21 août 2016)
} 
(centaines ou milliers d'années), mais elle n'a pas vocation à être appliquée à des individus. L'annonce du projet a soulevé un tollé chez les scientifiques et des objections de fond : déterminer l'appartenance du sujet à un groupe ethnique n'est pas pertinent pour connaitre sa nationalité, car les gens se déplacent, changent de pays, et de nombreuses frontières sont par ailleurs tracées sans préserver l'unité ethnique ${ }^{16}$. Le second volet de l'HPPP proposait une analyse isotopique des ongles et des cheveux des migrants. En effet les tissus humains sont affectés par l'alimentation et l'environnement. Un cheveu de quelques centimètres renferme donc une mémoire des conditions d'existence d'un sujet pendant les derniers mois et l'on peut éventuellement en déduire son parcours. Cette méthode est utilisée dans l'étude des migrations animales.

L'identification par le corps des nomades, des vagabonds des migrants est donc une longue suite de recherches, d'innovations et de perfectionnements. L'avènement du numérique, la modernisation de l'administration française, le traçage sur les réseaux, la géo localisation devraient logiquement mener la maîtrise du corps du migrant à la perfection. La réalité est plus nuancée. La période actuelle d'e-administration que connait la France conduit plutôt à une rupture entre le corps et l'identification administrative. Paradoxalement, il devient difficile de donner des preuves de filiation ou de présence sur un territoire.

\section{HOMO ADMINISTRATIVUS VS HOMO PHYSICUS}

Au-delà de son identité ou de son origine et selon les droits qu'il veut faire valoir, un immigré doit fournir des preuves de certains aspects de sa vie personnelle. Le recours à l'analyse corporelle montre alors ses limites pour répondre aux attentes de l'administration, comme le montrent les deux exemples suivants.

\footnotetext{
${ }^{16}$ Scientists Decry "Flawed" and "Horrifying" Nationality Tests, John Travis,Science,Sep. 29, 2009 , http://www.sciencemag.org/news/2009/09/scientists-decry-flawed-and-horrifying-nationality-tests (accédé le 15 juin 2016)
} 


\section{L'aporie du corps administré}

Quelques jours avant l'annonce du projet HPPP, le ministre de l'immigration français ${ }^{17}$ renonce à signer le décret d'application de la $1 \mathrm{li}^{18}$ sur les tests $\mathrm{ADN}$ pour le regroupement familial, mettant ainsi fin à une polémique de deux ans. Les principaux arguments opposés à ces tests, fiables à 99,9 \%, sont d'ordre éthique (le respect de la vie privée) et légaux (la proportionnalité des moyens d'investigation au regard du but poursuivi). Ils posent également la question du choix de société : la raison administrative peut-elle réduire la définition de la famille aux seuls liens biologiques en niant l'adoption, les recompositions familiales ou la prise en charge éducative ? Cependant, les tests ADN pour le regroupement familial restent pratiqués dans de nombreux pays d'Europe, parmi lesquels figurent la Belgique, la Suisse, l’Espagne ou le Royaume-Uni.

Il est parfois crucial pour un jeune réfugié de donner une preuve de son âge. En effet, mineur, il est confié à l'Aide Sociale à l'Enfance qui pourvoira à ses études et à sa subsistance ; majeur, il se voit invité à quitter le territoire national. Lorsqu'il ne détient pas d'acte de naissance, ou que ce document est mis en doute par l'autorité administrative, il peut être soumis à un test de maturité. L'évaluation de l'âge par le développement du système pileux a un temps été utilisé pour ce test, mais les jeunes le contournaient en s'épilant. Le test de maturité osseuse actuellement pratiqué, consiste en une radiographie de la main et du poignet que l'on compare à un référentiel, l'Atlas de de Greulich et Pyle, établi dans les années cinquante sur une population nord américaine de type caucasien. Invitée à se prononcer sur la validité du test dans le contexte des mineurs isolés étrangers, l'Académie de Médecine conclut en 2007 que « cette méthode ne permet pas de distinction nette entre seize et dix-huit ans ${ }^{19}{ }^{\prime}$. En 2016, le test est cependant toujours pratiqué en France, malgré son imperfection car il n'existe pas d'indice physiologique de la majorité. La

\footnotetext{
${ }^{17}$ Eric Besson.

${ }^{18}$ Il s'agit plus exactement, d'un amendement porté par le député Thierry Mariani voté par l'assemblée Nationale le 12 septembre 2007 et qui autorise aux agents diplomatiques et consulaires à proposer le recours aux tests génétiques, pour la délivrance de visas de longue durée «"En cas de doute sérieux sur l'authenticité de l'acte d'état civil". Cet amendement n'a, à l'heure où s'écrit cet article, jamais reçu de décret d'application.

${ }^{19}$ Bull. Acad. Natle Méd., 2007, 191, no 1, 139-142, séance du 16 janvier 2007 http://pe.sfrnet.org/Data/ModuleConsultationPoster/pdf/2007/1/f06f08b0-320e-4a7c-b13f-9a4ba4d4582e.pdf (accédé le 13 juin 2016)
} 
réalité physique, qui veut que la maturité s'installe progressivement, n'est pas réductible au concept légal de majorité, qui intervient à une date couperet. Entre renoncement stratégique et déni des évidences scientifiques, le recours de l'institution à l'indicialité corporelle relève du casse-tête politique.

\section{Vers une désincarnation administrative}

La logique administrative s'accommode donc de moins en moins bien du corps, variable, trompeur, manipulé, et se tourne vers la preuve documentaire. Ainsi en va-t-il pour la preuve de vie commune : le conjoint d'un(e) immigré(e) en situation régulière ou d'un(e) français(e) peut prétendre à un titre de séjour à condition de prouver la réalité d'une vie commune d'au moins dixhuit mois : le certificat de mariage ne suffit pas, pas plus que les enfants issus du couple ou les manifestations d'intimité (photos, lettres). Ce qui prouve le couple solidaire, pour la préfecture, c'est la réception régulière de courriers et de factures administrative portant les noms des deux membres du couple. Dans le même ordre d'idées, pour un père d'enfant français (qui peut solliciter pour ce motif un titre de séjour), la reconnaissance de l'enfant ou la production d'un certificat de naissance ne suffit pas. Venir en famille au bureau de la préfecture non plus. Ce qui « fait famille », ce sont les communautés d'adresses administratives, ou, à défaut, la trace, sur un compte bancaire, de versements réguliers prouvant que le père subvient aux besoins de la progéniture. Ainsi l'administration tente-t-elle de trouver une définition stable aux multiples formes familiales observables dans la réalité.

En droit français un migrant en situation illégale peut demander l'examen de sa situation pour être régularisé au bout de dix ans de présence effective et continue sur le territoire national. Venir en personne au guichet n'est donc pas suffisant puisqu'il faut prouver ce que le corps ne peut montrer: la mémoire de dix ans de présence. Là encore ce sont les traces administratives qui seront acceptées : factures de services publics, feuilles d'impositions, courriers recommandés. Ces documents doivent être soigneusement collectés dès l'arrivée sur le territoire, donc, dix ans avant la démarche. Si l'intéressé ne peut produire de documents administratifs pour certaines années il 
L'identification et le corps du migrant

sera supposé s'être absenté du territoire pour cette période, ce qui annule toutes les années antérieures de présence. L'administration électronique pourrait sécuriser ces dossiers en évitant les pertes dues à l'ignorance, la négligence ou aux sinistres, mais elle pose un autre problème : les démarches en ligne n'offrent plus aucune certitude sur la localisation «physique » de l'administré. En effet, même aujourd'hui, il subsiste un certain « face à face » aux bureaux des services publics. Le postier ou l'agent municipal ne connait pas l'usager mais, pour les démarches importantes, une pièce d'identité avec photo est exigée et lui permet de faire une relation entre l'identité annoncée et la personne qui lui fait face. Les traces administratives numériques, elles, sont totalement déconnectées du corps, puisque leur sécurisation repose sur un mot de passe, c'est-à-dire un procédé « par ce que l'on sait ».

\section{Les freins au data-panoptisme administratif}

Quelques ouvrages parmi les plus récents (Sadin, 2015 ; Mayer-Schönberger et Cukier, 2013 ; Bennett et al., 2014) alertent sur le risque que font courir au droit à la vie privée, l'utilisation des big data. Selon une idée largement répandue, nous produisons de multiples traces sur les réseaux, qui, collectées et inter-opérées, permettent de retracer une grande partie de nos activités. Paradoxalement, cela pourrait être une aubaine pour les « sans papiers » qui peinent à prouver la réalité de dix ans de présence sur le territoire français. Usagers de l'Etat comme les autres, ils prennent les transports en commun, consomment de l'électricité. Consommateurs, ils utilisent des cartes bancaires et des cartes de grands magasins, usent de téléphones portables et déposent ainsi sur les réseaux numériques publics et privés, d'innombrables traces géo-localisables. Il devrait être possible de consolider ces traces pour prouver l'ancienneté de leur résidence. Cela est vrai à cette nuance près que ce ne sont pas nos personnes physiques qui sont tracées, mais les dispositifs d'identifications numériques qui nous appartiennent : cartes à puces, mots de passe, mails, adresses ip des smartphones et des ordinateurs. Ils nous représentent par délégation, à condition que nous ne les prêtions pas ou qu'ils ne nous soient pas dérobés. En réalité ils n'offrent pas la 
certitude de notre identité effective, car, ce sont des preuves "par ce que l'on porte » qui n'intègrent pas de preuves « par ce que l'on est ».

\section{CONCLUSION : UNE PERIODE D'ENTRE DEUX A-CORPOREL}

Progressivement, l'identification quotidienne par les éléments corporels tend à se diluer dans la modernisation de 1'Etat. Ainsi en va-t-il aussi de la signature, qui intervient dans les procédés d'identification comme une empreinte du geste, le « tracé », qu'analysent les graphologues. Autrefois, les notaires appliquaient à leur signature boucles et arabesques complexes dont le tracé était difficile à imiter. Un expert graphologue lira dans le tracé manuel d'une écriture, ses déformations, la concordance avec l'âge de l'auteur supposé, son état de santé physique et moral, les circonstances de l'écriture. Pourtant intimement lié au corps, à l'identité et à la culture, le geste reste un élément administrativement peu exploré et probablement voué à l'oubli avec le développement $\mathrm{du}$ traitement de texte et de la signature électronique. Nous sommes donc aujourd'hui dans un entre deux, où la relation corps/identité semble complètement rompue. Des technologies sont susceptibles de la recréer : des puces électroniques glissées sous la peau, des ordinateurs qui ne démarrent qu'après reconnaissance de l'iris de l'œil ou de l'empreinte du pouce existent déjà. Il faudrait pour qu'ils délivrent une identité administrative fiable, que le législateur les rende obligatoires. Cela est possible, mais faut-il le souhaiter?

\section{Bibliographie}

BenNetT, C.J. (dir.), Vivre à nu: la surveillance au Canada, Au Press Athabasca University, 2014.

BERnARDOT, M., «A la recherche des sans-trace : cultures, espaces et citoyennetés », in L'Homme trace: perspectives anthropologiques des traces humaines contemporaines, Galinon-Mélénec, B.(dir.), Paris, CNRS Editions,2011, pp.331 -348. 
L'identification et le corps du migrant

Bertillon, A. , «De l'identification par les signalements anthropométriques. Conférence faite le 22 novembre au congrès pénitentiaire de Rome ", Archives de l'Anthropologie Criminelle, tome $1,1886$.

CoRAS, J. DE (1515-1572), Toulouse, F.P. de, 1572. Arrest mémorable du Parlement de Tholose : contenant une histoire prodigieuse d'un supposé mary,..., prononcé és Arrestz généraux le XII septembre 1560 ([Reprod.]) < http://gallica.bnf.fr/ark:/12148/bpt6k52469j>.

CuEnOD, C. , «Une signalétique accusatoire : les pratiques d'identification judiciaire au XVIIIe siècle ». Crime, Histoire \& Sociétés / Crime, History \& Societies, ${ }^{\circ} 12,2018$, pp. 5-31

DENIS, V. , «Administrer l’identité », Labyrinthe, n5, 2000, pp. 25-42

Diamant-Berger, O. , Nauwelaers, J. « Détermination médico-légale de l'âge du sujet jeune, dossier «"Le mineur étranger en exil " » diffusé au XIème colloque de droit des étrangers du Syndicat des Avocats de France (22 mars 2003, Lille)

Foucault, M. Surveiller et punir : naissance de la prison, Paris, Gallimard, 1975.

Fraenkel, B. , «La signature : du signe à l'acte », Sociétés \& Représentations Vol.25, n¹3, 2008.

FraEnKel, B. , La Signature: Genèse d'un signe, Paris, Gallimard, 1992.

GALinOn-MelenEC, B. (dir.), L'Homme trace: perspectives anthropologiques des traces humaines contemporaines, Paris, CNRS Editions, 2011.

GALINON-Melenec, B. ET AL (dir.), L'Homme trace. Inscriptions corporelles et techniques, Paris, CNRS Editions, 2015.

GramaCciA, G., «Les institutions de la médiation symbolique en quête de l'Homme-trace », in L'Homme trace: perspectives anthropologiques des traces humaines contemporaines, GalinonMélénec, B.(dir.), Paris, CNRS Editions, 2011, pp.273 -287).

GutTon, J.P., L'État et la mendicité dans la première moitié du XVIIIe [dix-huitième] siècle, Auvergne, Beaujolais, Forez, Lyonnais. Université de Saint-Etienne, 1973. 
LACHIVER, M. , «Une source méconnue pour l'étude de la mobilité géographique en France au XVIIIe siècle. Les congés et translations de domicile », Population $n^{\circ} 32,1977$, pp. 353-373

MAtTelart, A., La globalisation de la surveillance : aux origines de l'ordre sécuritaire, Paris, La Découverte, 2007.

MAYer-SchÖnberger, V. et CuKIER, K., Big Data : la révolution des données est en marche, Paris, Robert Laffont, 2014.

NoIRIEL, G. , Population, immigration et identité nationale en France (xix ${ }^{e}-x x^{e}$ siècle), Paris, Hachette, coll. « Carré-Histoire » 1992

NoIRIEL, G. , L'identification. Genèse d'un travail d'état, Paris, Belin, 2007

PIAZZA, P. , « Du bertillonnage à l'Europe biométrique », in: Identification et Surveillance Des Individus: Quels Enjeux Pour Nos Démocraties ?, Paris, Éditions de la Bibliothèque publique d'information, 2014, pp. 13-25.

REISS, R.A. Manuel du portrait parlé (méthode Alphonse Bertillon) à l'usage de la police : avec vocabulaire français, allemand, italien et anglais , Paris, Schlachter, 1905.

ReYnAUD, V., Une histoire de l'identité, Seyssel, Champ Vallon, 2008.

SADIN, E., La vie algorithmique : Critique de la raison numérique. Paris, L’Echappée, 2015.

Tessier, G., « Diplomatique », in L’Histoire et ses méthodes, Paris, Gallimard, (Encyclopédie de la Pléiade), 1961 
L'identification et le corps du migrant

Illustration

Fig.1 Frontispice de L’identification anthropométrique : instructions signalétiques, Alphonse Bertillon, 1893

\section{RELEVÉ \\ D U}

\section{SIGNALEMENT ANTHROPOMÉTRIQUE}

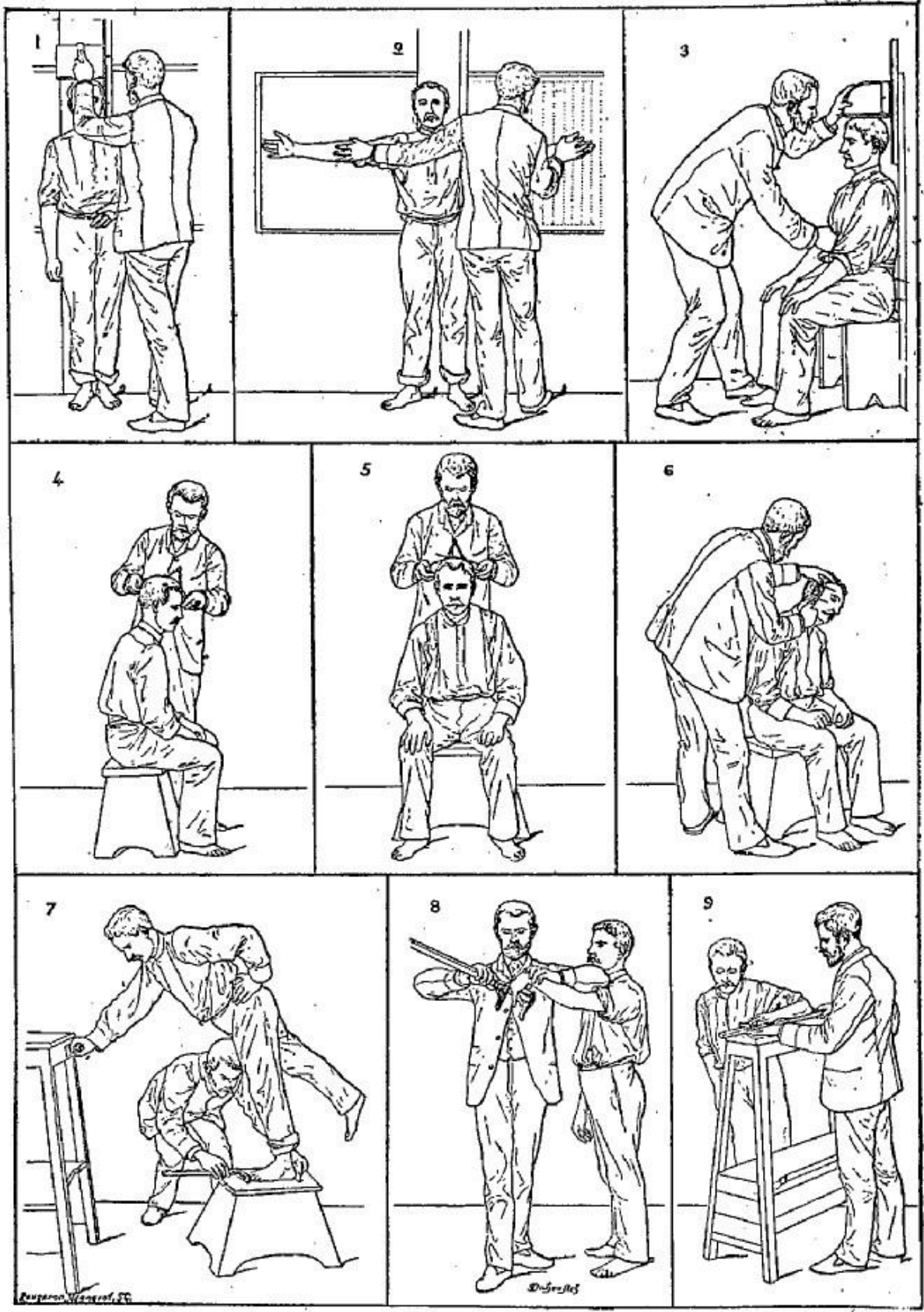

x. Taille. - 2. Envergure. - 3. Buste. -

4. Longueur de la tète. -5 . Largeur de la tête. -6 . Oreille droite. 7. Pied gauche -8 . Médius gauche: -9 . Coudée gauche. 